\title{
Control Experimental del Modelo de Péndulo Matemático*
}

(Experimental control of mathematical pendulum model)

\author{
César Medina ${ }^{1}$, Sandra Velazco ${ }^{1}$, Julia Salinas ${ }^{1,2}$ \\ 1 Universidad Nacional de Tucumán \\ Av. Independencia 1800, (4000) San Miguel de Tucumán \\ 2 CONICET \\ jsalinas@herrera.unt.edu.ar
}

Recebido em 28 de novembro, 2001. Aceito em 15 de janeiro, 2002.

\begin{abstract}
Se aportan consideraciones sobre un trabajo práctico de laboratorio de Física destinado a alumnos con conocimientos teóricos sobre oscilaciones. Se propone a los estudiantes construir un péndulo que se comporte como matemático, y realizar un análisis del error introducido por los supuestos del modelo. Entre ellos, se analizan cuantitativamente: rozamiento despreciable, amplitud pequeña, hilo inextensible, masa puntual del cuerpo, masa despreciable del hilo. Se concluye que los supuestos del modelo se pueden cumplir fácilmente en un caso práctico, y que su análisis permite que los estudiantes adquieran una mejor comprensión del modo científico de controlar la validez de los desarrollos teóricos y valoren la importancia y la utilidad de los modelos sencillos en la ciencia.
\end{abstract}

Considerations about a Physics laboratory experiment intended to students with some theoretical knowledge about oscillations are given. Students are proposed to construct a pendulum that behaves as a mathematical one, and to analyze the error due to the model assumption. Among these hypothesis, the following ones are quantitatively analyzed: vanishing friction, small amplitude, inextensible wire, punctual mass of the body, vanishing mass of the wire. It is concluded that model hypothesis are easily accomplished in practice, and that their analysis allow a better students'comprehension of the scientific way to control theoretical development validity

\section{Introducción}

Los referentes directos de las teorías científicas no son los fenómenos naturales (inabordables en su complejidad global) sino los modelos, es decir, construcciones intelectuales basadas en generalizaciones, abstracciones e idealizaciones (Bunge 1985).

En particular, los modelos sencillos, además de su importancia científica, constituyen una valiosa herramienta didáctica mediante la cual el alumno puede involucrarse en actividades y tomas de decisión coherentes con las desarrolladas por la comunidad científica, y controlar la adecuación entre teoría y realidad.

\section{Planteo del Problema}

Lo que sigue aporta consideraciones en el desarrollo de un trabajo práctico de laboratorio orientado a alumnos que ya posean conocimientos teóricos sobre oscila- ciones mecánicas. Entre los diversos textos que tratan este tema y sus complementarios con el nivel adecuado, elegimos para facilidad del lector, basar las referencias bibliográficas en uno bastante conocido: Física, Vol I, de Resnick, Halliday y Krane, 1993 (en adelante citado como R-H-K).

En particular, el estudiante debe conocer las ecuaciones del período de a) un péndulo físico, es decir, cualquier cuerpo rígido montado de manera que pueda oscilar en un plano vertical, respecto a algún eje que pase por él, y $b$ ) de un péndulo matemático, es decir, una partícula suspendida de un cordón ligero inextensible (R-H-K, cap.15, §5):

a) Período de un péndulo físico:

$$
T_{f}=2 \pi \sqrt{\frac{I}{m \cdot g \cdot d}}
$$

\footnotetext{
*Una versión previa de este trabajo ha sido presentado en el Encuentro Nacional de Profesores de Física, (Córdoba, Argentina, 22 al 24 de mayo de 2001) y aparece en las Memorias de la Reunión.
} 
donde $T$ representa el período; $I$, el momento de inercia del cuerpo; $m$, la masa del cuerpo; $g$, el valor local de la aceleración de la gravedad, y $d$, la distancia entre el eje de giro y el centro de gravedad del sistema.

b) Período de un péndulo matemático:

$$
T_{m}=2 \pi \sqrt{\frac{l}{g}}
$$

donde $l$ representa la longitud del hilo.

Se propone a los estudiantes que construyan un péndulo que se comporte como matemático y que, a partir de la medición de su período, determinen el valor local de la aceleración de la gravedad, $g$, con un error prefijado; usualmente dado en términos relativos: $\varepsilon=\Delta g / g$. Para ello, deberán realizar un análisis cuantitativo de los errores introducidos por el proceso de medición y por las idealizaciones del modelo usado.

Este trabajo está centrado en el control del error introducido por el alejamiento de las condiciones impuestas por el modelo de péndulo matemático. Enunciemos, pues, brevemente estas condiciones, y luego analizaremos los errores que introduce la falta de su cumplimiento estricto.

La ec. (1) se deduce suponiendo:

$\mathrm{S}_{1}$ : Rozamiento despreciable (en la ec. de movimiento, la fuerza total aceleradora es, en cada instante, la componente tangencial del peso del cuerpo).

$\mathrm{S}_{2}$ : Amplitudes pequeñas de oscilación (en la ecuación de movimiento, el seno del ángulo de amplitud puede ser reemplazado por el ángulo mismo).

$\mathrm{S}_{3}$ : Un cuerpo rígido (distribución invariable de masa y, por tanto, momento de inercia constante).

Por su parte, la ec. (2) es un caso particular de la (1), cuya validez debe controlarse para un sistema como el construido por los estudiantes. Luego, la (2) debe cumplir los mismos supuestos que la (1) más otros adicionales.

Para considerar al péndulo matemático como un caso particular del péndulo físico, cabe en primer lugar, reformular el supuesto $\mathrm{S}_{3}$, ya que un hilo no puede considerarse un cuerpo rígido. Sin embargo, la distribución de masas del péndulo sí se puede considerar invariable si el hilo no cambia su longitud a lo largo del movimiento.

Así, $\mathrm{S}_{3}$ queda:

$\mathrm{S}_{3}$ : el hilo debe ser inextensible.

Los supuestos adicionales se basan en dos requisitos que permiten pasar de la ec. (1) a la (2). Éstos están asociados a una expresión sencilla para el momento de inercia, y a la condición de que $d$ sea igual a $l$. Para ello, se debe cumplir:
$\mathrm{S}_{4}$ : La masa del cuerpo debe ser puntual.

$\mathrm{S}_{5}$ : La masa del hilo debe ser despreciable frente a la del cuerpo.

En efecto, bajo estas condiciones:

$$
\begin{aligned}
T & =2 \pi \sqrt{\frac{I}{m \cdot g \cdot d}} \\
& =2 \pi \sqrt{\frac{m_{p} \cdot l^{2}}{m_{p} \cdot g \cdot l}} \\
& =2 \pi \sqrt{\frac{l}{g}}
\end{aligned}
$$

\section{Análisis del Error Intro- ducido por los Supuestos del Modelo}

\section{Rozamiento Despreciable}

El efecto de rozamiento puede darse entre el sistema y el medio en el cual oscila, y entre el hilo y el eje de oscilación. Este último es más fácil de evitar que de evaluar matemáticamente, por lo cual se recomienda fijar el hilo al eje de una forma lo suficientemente firme como para evitar cualquier desplazamiento de la ligadura. En particular se debe evitar "enrollar" el hilo o hacer ligaduras en forma de "anillo" suelto. Si se lo enrolla, el hilo, a lo largo de la oscilación, estará enrollándose y desenrollándose una porción de vuelta, lo cual variará su longitud efectiva a lo largo de cada oscilación. Si por otra parte, a la ligadura se le da la forma de un anillo suelto se introducirá un rozamiento difícil de evaluar.

Para evaluar el rozamiento con el aire, consideremos el torque (R-H-K, cap.12, §4 ) que éste ejerce sobre el sistema:

$$
M_{R}=F_{R} \cdot d
$$

donde $d$ representa el brazo de palanca efectivo, y $F_{R}$, la fuerza de roce. Ambos factores dependen de la forma y tamaño del cuerpo, y $F_{R}$, además, de la viscosidad del aire y de la velocidad del cuerpo relativa al aire. Como se está trabajando con cuerpos pequeños, cabe suponer, en primera aproximación, que $d$ es la distancia entre el eje de oscilación y el centro de gravedad del cuerpo.

Para velocidades pequeñas (R-H-K, cap.15, §8),

$$
F_{R}=b \cdot v
$$


siendo $b$ la constante de rozamiento entre el aire y el cuerpo; y $v$, la velocidad del cuerpo relativa al aire. Incluyendo la ec. (4) en la ec. diferencial de movimiento, se obtiene (R-H-K, cap.15, §8)

$$
\alpha=\alpha_{o} \cdot e^{\frac{-b t}{2 m}} \cdot \cos \left(\omega^{\prime} t+\varphi\right)
$$

donde la exponencial representa el efecto de amortiguamiento, $\omega$ ' es la frecuencia del movimiento amortiguado y $\varphi$ es el ángulo de fase.

Así, para un péndulo matemático, el período del movimiento amortiguado queda

$$
\begin{aligned}
T^{\prime} & =2 \pi / \omega^{\prime} \\
& =\frac{2 \pi}{\sqrt{\frac{g}{t}-\left(\frac{b}{2 m}\right)^{2}}}
\end{aligned}
$$

De la (7) se deduce que para que el roce del aire se pueda considerar despreciable, el segundo término en el discriminante de la raíz debe ser muy pequeño frente al primero, como efectivamente lo es, por los valores que pueden asumir las variables.

Analicemos esto cuantitativamente. El error relativo en el período introducido por usar la ec. (2) en lugar de la (7) es

$$
\frac{\Delta T}{T}=\frac{T^{\prime}-T_{m}}{T_{m}}=\frac{\frac{2 \pi}{\sqrt{\frac{g}{l}-\left(\frac{b}{2 m}\right)^{2}}}-\frac{2 \pi}{\sqrt{\frac{g}{l}}}}{\frac{2 \pi}{\sqrt{\frac{g}{l}}}}
$$

La ec. (8), luego de dividir numerador y denominador en $2 \pi(g / l)^{-1 / 2}$, queda

$$
\frac{\Delta T}{T}=\left(1-\frac{(b / 2 m)^{2}}{g l}\right)^{-1 / 2}-1
$$

y desarrollando el paréntesis del segundo miembro en serie binomial, hasta el segundo término, se obtiene

$$
\frac{T-T_{m}}{T_{m}}=\frac{1}{2} \frac{(b / 2 m)^{2}}{g / l}
$$

Luego, basta que el segundo miembro de la ec. (10) sea menor que el error prefijado $\varepsilon$ para que el efecto del amortiguamiento sea despreciable.

\section{Amplitud Inicial Pequeña}

La ecuación para el período del péndulo matemático que incluye la dependencia con la amplitud es (R-H-K, cap. $15, \S 5$, y sus referencias):
$T_{\alpha}=2 \pi \sqrt{\frac{l}{g}}\left(1+\frac{1^{2}}{2^{2}} \operatorname{sen}^{2}(\alpha / 2)+\frac{1^{2} \cdot 3^{2}}{2^{2} \cdot 4^{2}} \operatorname{sen}^{4}(\alpha / 2)+\ldots\right)$

que, por propiedades de convergencia, se puede truncar en cualquier término obteniéndose que la suma hasta ese término será mayor que la suma de los restantes.

Así, truncándola en el segundo, a los fines de calcular el error, se tiene

$$
T_{\alpha}=2 \pi \sqrt{\frac{l}{g}}\left(1+\frac{1^{2}}{2^{2}} \operatorname{sen}^{2}(\alpha / 2)\right)
$$

Luego, el error relativo introducido por usar la ec. (2), no dependiente de la amplitud, en lugar de la (12), dependiente de la amplitud es

$$
\frac{\Delta T}{T}=\frac{T_{\alpha}-T_{m}}{T_{m}}=\frac{1^{2}}{2^{2}} \operatorname{sen}^{2}(\alpha / 2)
$$

de donde se puede calcular $\alpha$, luego de igualar el segundo miembro a un valor menor que el error prefijado $\varepsilon$.

\section{Hilo Inextensible}

Para analizar esta condición debemos considerar dos aspectos: i) la deformación del hilo al aplicar el peso del cuerpo en forma estática, y ii) la deformación del hilo producida durante la oscilación.

Al aplicar el peso del cuerpo en forma estática, el hilo puede sufrir una elongación considerable sin que esto afecte el modelo. Basta con que la deformación no supere el límite elástico y la densidad lineal permanezca constante, a fin de calcular el momento de inercia.

Durante la oscilación, debe tenerse presente que el hilo está sometido a una tensión variable. Su módulo es igual a la suma de la componente radial del peso más la fuerza centrípeta asociada al movimiento circular (RH-K, cap.4, §5),

$$
\tau=P \cdot \cos \alpha+m \cdot \frac{v}{l}^{2}
$$

donde $P$ representa el peso del cuerpo y v su velocidad respecto al aire.

Igualando la energía cinética máxima del cuerpo a su energía potencial máxima (R-H-K, cap.7, §4), se puede calcular la velocidad del cuerpo al pasar por la posición de equilibrio:

$$
v=\sqrt{2 \cdot g \cdot l\left(1-\cos \alpha_{0}\right)}
$$


El hilo sufrirá una tensión mínima cuando esté en su máxima amplitud y una máxima cuando pase por su posición de equilibrio. La diferencia entre estos valores extremos es

$$
\Delta \tau=3 P\left(1-\cos \alpha_{o}\right)
$$

Luego, una vez fijada la amplitud inicial según la ec. (13), se debe elegir un hilo lo suficientemente resistente a la tracción (ya sea por su módulo de Young y/o por sus dimensiones), o un cuerpo lo suficientemente liviano para que la diferencia de tensión de la (16) lo elongue una cantidad menor que la fijada para el error de su longitud. En este sentido es recomendable no usar hilos vinílicos ni de fibras textiles, excepto con cuerpos livianos y amplitudes pequeñas.

\section{Masa Puntual}

La distribución de masa del cuerpo afecta al período del péndulo a través del momento de inercia. Aplicando el teorema de los ejes paralelos (Steiner), se observa que dicho momento de inercia está dado por (R-H-K, cap.12, §2)

$$
I_{p}=I_{G}+m_{p} \cdot d^{2}
$$

donde $I_{G}$ es el momento de inercia del cuerpo respecto de su centro de gravedad, $m_{p}$ es la masa del cuerpo, y des la distancia desde el centro de gravedad del cuerpo hasta el eje de oscilación.

Dado que el segundo término del segundo miembro de (17) es el momento de inercia de una masa puntual, el primer término puede interpretarse como el error en exceso debido a que la masa del cuerpo no lo es. Luego, basta con que el cociente entre el primer término y el segundo sea suficientemente pequeño para que la masa pueda considerarse puntual, sin importar las dimensiones del cuerpo.

Para saber cuán pequeño debe ser este cociente, consideremos el error relativo introducido por usar el período del péndulo matemático en lugar del de un péndulo físico, $T_{G}$. Suponemos que el péndulo físico está construido con un hilo de masa despreciable pero con un cuerpo de tamaño finito. Por otra parte, para el período del péndulo matemático, por simplicidad, usaremos "d" en lugar de "l", que en este sistema son iguales. Luego,

$$
\begin{gathered}
\frac{\Delta T}{T}=\frac{T_{G}-T_{m}}{T_{m}}=\frac{2 \pi \sqrt{\frac{I_{G}+m_{p} \cdot d^{2}}{m_{p} \cdot g \cdot d}}-2 \pi \sqrt{\frac{d}{g}}}{2 \pi \sqrt{\frac{d}{g}}} \\
=\frac{2 \pi \sqrt{\frac{d}{g}\left(\frac{I_{G}}{m_{p} \cdot d^{2}}+1\right)}-2 \pi \sqrt{\frac{d}{g}}}{2 \pi \sqrt{\frac{d}{g}}} \\
=\left(\frac{I_{G}}{m_{p} \cdot d^{2}}+1\right)^{1 / 2}-1
\end{gathered}
$$

Haciendo el desarrollo binomial, hasta el segundo término del paréntesis del último miembro, se obtiene que para que la masa del cuerpo pueda considerarse puntual basta que

$$
\frac{1}{2} \frac{I_{G}}{m_{p} \cdot d^{2}}<<\varepsilon
$$

\section{Hilo de Masa Despreciable}

La masa del hilo afecta el período por tres motivos:

I) porque contribuye al momento de inercia del sistema:

$$
I=I_{\text {hilo }}+I_{\text {pesa }}
$$

Dado que aumenta $I$, en comparación con el péndulo matemático (ec. (3)), este factor tiende a aumentar el período.

II) porque cambia la posición del centro de gravedad, cuya distancia al eje de oscilación será (RH-K, cap.14, §2)

$$
d=\frac{m_{h} \cdot l / 2+m_{p} \cdot l}{m_{h}+m_{p}}
$$

Dado que disminuye $d$, en comparación con el péndulo matemático (ec. (3)), este factor también tiende a aumentar el período.

III) porque cambia la masa total del sistema:

$$
m=m_{\text {hilo }}+m_{\text {pesa }}
$$

Dado que aumenta $m$, en comparación con el péndulo matemático (ec. (3)), este factor tiende a disminuir el período.

El momento de inercia del hilo -que debe reemplazarse en la ec. (20)- si su densidad lineal es homogénea, está dado por (R-H-K, cap.12, §4)

$$
I_{\text {hilo }}=\frac{1}{3} \cdot m_{\text {hilo }} \cdot l^{2}
$$


Reemplazando (20), (21), (22) y (23) en (1), y definiendo

$$
k=m_{h} / m_{p}
$$

se puede calcular cuánto se aparta el modelo de péndulo matemático del modelo de péndulo físico:

$$
\begin{aligned}
2 \pi & \sqrt{\frac{I}{m \cdot g \cdot d}}=2 \pi \sqrt{\frac{I_{h}+I_{p}}{\left(m_{h}+m_{p}\right) \cdot g \cdot \frac{m_{h} \cdot 1 / 2+m_{p} \cdot 1}{m_{h}+m_{p}}}} \\
& =2 \pi \sqrt{\frac{\left(\frac{1}{3} k+1\right) \cdot m_{p} \cdot l^{2}}{g \cdot\left(\frac{1}{2} k+1\right) \cdot l \cdot m_{p}}} \\
& =2 \pi \sqrt{\frac{l}{g}} \cdot \sqrt{\frac{\left(\frac{1}{3} k+1\right)}{\left(\frac{1}{2} k+1\right)}}
\end{aligned}
$$

El último coeficiente del último miembro es obviamente menor que la unidad. Esto indica que el aumento de la masa total prevalece sobre los otros dos factores arriba analizados en relación a la masa del hilo. Luego, se puede escribir

$$
\begin{aligned}
& \frac{\Delta T}{T}=\frac{T_{h}-T_{m}}{T_{m}} \\
& =\frac{T_{m} \sqrt{\frac{\left(\frac{1}{3} k+1\right)}{\left(\frac{1}{2} k+1\right)}}-T_{m}}{T_{m}} \\
& =\sqrt{\frac{\left(\frac{1}{3} k+1\right)}{\left(\frac{1}{2} k+1\right)}}-1
\end{aligned}
$$

Obsérvese en el último miembro que, por ser el primer término menor que 1 , el error relativo debido a la masa no nula del hilo,.es negativo (por defecto).

\section{Conclusiones}

Del análisis cuantitativo del error se deduce que las hipótesis del modelo pueden cumplirse fácilmente en un caso práctico. Por otra parte, expresiones como "amplitud pequeña", "masa puntual", "hilo inextensible", "masa despreciable", pueden adquirir significados muy distintos de los que los alumnos podrían atribuirles basados en interpretaciones literales, definiciones previas, o conceptos construidos en otros contextos.

Así, dentro de un error del 1\%, en cada caso (por separado), una amplitud inicial de $23^{\circ}$ es pequeña; una esfera cuyo diámetro es el $30 \%$ de la longitud del hilo, es puntual; la masa de un hilo, igual al $10 \%$ de la masa de la pesa, es despreciable; el hilo puede sufrir una elongación arbitraria, dentro de su límite elástico, al ser cargado estáticamente o bien la tensión a la que está sometido puede variar durante la oscilación, sin dejar de ser "inextensible".

El desarrollo de este práctico permite que los estudiantes adquieran una mejor comprensión del modo científico de controlar la validez de los desarrollos teóricos y valoren la simplicidad como una condición deseable y útil de los modelos científicos.

\section{Bibliografia}

[1] Bunge, M. 1985. La Investigación Cientifica, Editorial Ariel, Barcelona.

[2] Halliday, D., Resnick, R., Krane, K. 1993. Física, Vol 1, $4^{a}$ ed. Compañía Editorial Continental, México. 